\title{
Plasmonic resonances in copper island films
}

\author{
Jordi Sancho-Parramon, Boris Okorn, Krešimir Salamon, Vesna Janicki* \\ Ruđer Bošković Institute, Bijenička cesta 54, Zagreb, 10000, Croatia
}

\begin{abstract}
The optical properties of $\mathrm{Cu}$ island films, in particular their plasmonic behaviour, are investigated. The films are fabricated by electron beam evaporation using different deposition parameters (deposited mass thickness and substrate temperature). The optical response can be tuned from a metal-like behaviour to well-defined localized surface plasmon resonances and is well correlated with the structural and morphological properties of the samples. Significant changes of optical properties take place with sample aging, including quenching and non-monotonic frequency shift of the plasmon resonance. These changes are interpreted in terms of $\mathrm{Cu}$ oxidization process, that appears to strongly depend on the initial morphology of samples. Theoretical calculations in the framework of effective medium theories qualitatively explain the experimental observations. Overall, the results give a detailed insight on the morphology dependence and time evolution of the optical response of $\mathrm{Cu}$ island films that may provide useful guidelines for applying these nanostructures in plasmonic applications.

Keywords: copper, metal island films, localized surface plasmon resonance, optical characterization, ellipsometry, copper oxide, effective medium theory
\end{abstract}

\footnotetext{
${ }^{*}$ Corresponding author. E-mail: janicki@irb.hr
} 


\section{Introduction}

Over the last two decades, advances in nanoscale fabrication and simulation techniques have enabled to envisage novel applications based on the surface plasmon resonance phenomenon, giving birth to plasmonics as a research field of its own $[1,2]$. Ideally, plasmonics requires using materials with a negative dielectric function and negligible losses [3] and hence Ag [4] is the preferred choice for design of devices and nanostructures operating in the visible range. Since material stability is an important factor for most applications, $\mathrm{Au}$ is often a more practical option because it is less reactive than Ag. Nevertheless, Au shows poor plasmonic performance in the spectral range below $600 \mathrm{~nm}$ due to the presence of interband transitions [5]. Hence, there is an on-going quest for alternative plasmonic materials investigating properties of other pure metals, nitrides, oxides, silicides and 2D materials, among others $[3,6]$.

Copper is potentially a good candidate for plasmonics, but it easily oxidizes in normal atmosphere forming $\mathrm{Cu}_{2} \mathrm{O}$ and $\mathrm{CuO}$ [7]. Measurements on oxide-free nanoparticles evidence plasmon resonances comparable to those of $\mathrm{Ag}$ and $\mathrm{Au}$ [8]. Recently, coating of $\mathrm{Cu}$ nanoparticles with thin $\mathrm{Al}_{2} \mathrm{O}_{3}$ [9] or graphene overlayers [10] and treatments with corrosion inhibitors [11] have shown to largely improve their chemical stability and hence the plasmonic response. Furthermore, the presence of an oxide shell with specific thickness values may actually enhance the localized surface plasmon resonance of $\mathrm{Cu}$ rather than quenching it [12]. In addition to chemically synthesized nanoparticles, ultrathin compact films deposited on dielectric substrates can show plasmonic resonances due to the presence of metal islands that result from the Vollmer-Weber film growth mechanism [13]. As the amount of deposited metal increases, the islands become closer and eventually percolate, resulting into tuning of the optical response from a pure plasmonic resonance to a metal-like behaviour 
[14]. This tunability has been exploited to obtain materials with unusual effective optical response that can improve or offer new optical coating functionalities [15].

In this study we investigate the optical properties of $\mathrm{Cu}$ metal island films deposited by electron beam evaporation and characterized by means of several optical and structural measurement techniques (spectroscopic ellipsometry, spectrophotometry, $\mathrm{X}$-ray diffraction, scanning electron microscopy). The results evidence the possibility to broadly tune the optical response of $\mathrm{Cu}$ island films by changing the deposited mass thickness and substrate temperature. The time evolution of the samples optical properties is correlated with the oxidization of the films, that strongly depends on the initial morphology of the sample. The results help elucidating the role of morphology and oxidization on the optical response of $\mathrm{Cu}$ island films and provide new insights that might be useful for effectively incorporating $\mathrm{Cu}$ nanostructures in plasmonics applications.

\section{Materials and methods}

$\mathrm{Cu}$ island films were deposited on silicon and BK7 glass substrates by electron beam evaporation using a modified Varian chamber with base pressure of $6 \times 10^{-7}$

Torr. A quartz crystal was used to monitor the deposited mass thickness $\left(d_{\text {mass }}\right)$, that was varied between 2 and $12.5 \mathrm{~nm}$. The deposition rate of $\mathrm{Cu}$ was $\approx 1 \AA / \mathrm{s}$. Substrates were pre-heated at different temperatures $\left(T_{s}\right)$. Both kind of substrates were pre-coated with a $80 \mathrm{~nm}$ thick $\mathrm{SiO}_{2}$ layer in order to insure the same growth conditions for $\mathrm{Cu}$ island growth. Samples deposited on glass substrates were used for transmittance and ellipsometric measurements while those deposited on Si were used for scanning electron microscopy and X-ray diffraction.

Transmittance measurements were taken in the range between 1.12 and $4 \mathrm{eV} \mathrm{nm}$ using a Lambda 25 Perkin-Elmer spectrophotometer. Measurements were performed 
just after the samples were taken out from the deposition chamber and later at different time intervals in order to monitor changes in the optical response upon exposition to normal atmosphere. Spectroscopic ellipsometry measurements were carried out on samples deposited on BK7 substrates in the spectral range between 0.57 and $4.6 \mathrm{eV}$ and angles of incidence of $45^{\circ}, 55^{\circ}$ and $65^{\circ}$ with a J.A. Woollam V-VASE ellipsometer. Modeling of ellipsometric data lead to retrieval of the films effective thickness $\left(d_{e f f}\right)$ and dielectric function (see Section Theory). Ellipsometric measurements were taken one day after sample deposition and after two months.

$\mathrm{X}$-ray diffraction (XRD) measurements were performed on selected samples in or-

der to verify the presence of copper oxide in the films. Measurements were carried out in a diffractometer equipped with a Co X-ray tube and under fixed grazing incidence angle ensuring the maximum diffraction yield from thin films. Scanning electron microscopy (SEM) plain view images were taken with a field-emission microscope Jeol JSM 7000F, typically working with an acceleration voltage of $15 \mathrm{kV}$.

\section{Theory}

\subsection{Maxwell-Garnett effective medium approximation for metal island films}

An accurate computation of the electromagnetic response of metal island films is a cumbersome task due to irregular particle shape, particle size distribution, electromagnetic coupling among islands and possible intrinsic size effects. Nevertheless, taking into account that the typical dimensions of metal islands are much smaller than the wavelength of light used in the measurements, the film optical response can be well described in terms of an effective dielectric function $\left(\epsilon_{e f f}\right)$. Classical effective medium theories generally overlook the morphological complexity of the films [16], yet they provide a good qualitative description of their optical behavior [17, 18, 19]. 
In these cases, metal island films are modeled as an ensemble of $\mathrm{Cu}$ ellipsoids with dielectric function $\epsilon_{C u}$ embedded in a host medium with dielectric function $\epsilon_{h}$ and occupying a fraction $f$ of the film volume. Then, the effective dielectric function in the framework of the Maxwell-Garnett approximation, $\epsilon_{M G}$, reads as [20]:

$$
\epsilon_{M G}=\frac{\epsilon_{h}+\left[L_{p}(1-f)+f\right]\left(\epsilon_{C u}-\epsilon_{h}\right)}{\epsilon_{h}+L_{p}(1-f)\left(\epsilon_{C u}-\epsilon_{h}\right)}
$$

where $L_{p}$ are the depolarization factors of the particles, that are calculated in terms of the three ellipsoid semi-axes $(a, b, c)$ [21]:

$$
L_{p}=\frac{a b c}{2} \int_{0}^{\infty} \frac{d q}{\left(\chi_{p}^{2}+q\right) f_{k}(q)}
$$

where $\chi_{p}$ takes the values $a, b, c$ for incoming electric fields parallel to the correspond-

ing axes and $f_{k}(q)=\left[\left(a^{2}+q\right)\left(b^{2}+q\right)\left(c_{2}+q\right)\right]^{1 / 2}$. In first approximation, particles in metal island films can be modeled as flattened ellipsoids with the short semi-axis (a) perpendicular to the sample surface $[17,18,19]$. In the following we consider the response of the system to electric fields applied parallel to the sample surface, i.e., to the $b$ and $c$ semi-axes, as it corresponds to near-normal incidence measurements. Although more advanced models for metal island films have been developed [22], Equation 1 is able to take into account the main features describing the localized surface plasmon resonance dependence with the sample morphology.

\subsection{Dispersion model for ellipsometric data analysis}

As mentioned above, classical effective medium theories provide a qualitative description of the optical response of metal island films but are unable to accurately fit experimental data. Previous studies have shown that a multiple oscillator model can adequately represent the actual effective dielectric function of this kind of samples $[23,24,25,26]$. Accordingly, we use the following dispersion model: 


$$
\epsilon_{e f f}(E)=\epsilon_{\infty}-\frac{\omega_{p}^{2}}{E(E+i \gamma)}+\sum_{k=1}^{n}\left[\epsilon_{G R, k}(E)+i \epsilon_{G I, k}(E)\right]
$$

with

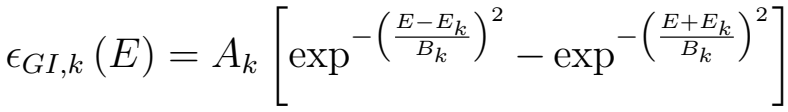

$$
\begin{aligned}
& \epsilon_{G R, k}(E)=\frac{2}{\pi} p \cdot v \cdot \text { Cauchy } \int_{0}^{\infty} \frac{\xi \epsilon_{G I, k}(\xi)}{\xi^{2}-E^{2}} d \xi
\end{aligned}
$$

where $E$ is the photon energy. The first term on the right-hand side of Eq. $3, \epsilon_{\infty}$, is the contribution of polarization mechanisms that occur at photon energies above the spectral range of interest. The second term is the Drude model that describes the metal-like behavior of percolated films and is defined through the plasma frequency $\left(\omega_{p}\right)$ and the damping constant $(\gamma)$. Finally, the last term is a sum of Gaussian oscillators that can be used to model localized surface plasmon resonance and interband transitions. This type of oscillators is more appropriate than the classical Lorentz model in the case of complex systems like metal island films, in which the the size and shape distribution of particles often results into a nearly Gaussian distribution of resonances $[23,26]$. Each Gaussian oscillator is defined through its amplitude $\left(A_{k}\right)$, central energy $\left(E_{k}\right)$ and broadening $\left(B_{k}\right)$. Usually, one to two oscillators are necessary to describe the plasmonic resonances and one additional oscillator is required for each interband transition appearing in the measured spectral range. Experimental data fitting was carried out using the WVASE software and lead to the optimal values of the dispersion model parameters by minimizing the discrepancy between measured and simulated data. 


\section{Results and discussion}

\subsection{Influence of deposition conditions}

First, we analyze the variation of the samples optical properties with $d_{\text {mass }}$. Fig. 1 shows the real and imaginary part of $\epsilon_{e f f}$ of films deposited keeping the substrate at room temperature (RT) and having different mass thickness (5, 8 and $12.5 \mathrm{~nm})$. Bulk $\mathrm{Cu}$ optical constants determined by Johnson and Christy are included for comparison [5]. In all the cases, the $\mathrm{Cu}$ island films show metal-like response, as the Drude term had to be included in Eq. 3 in order to obtain satisfactory data fits. No identifiable plasmon resonance signature is observed, except for the sample with $d_{\text {mass }}=5 \mathrm{~nm}$, that shows a weak shoulder in the imaginary part of $\epsilon_{\text {eff }}$ at $1.1 \mathrm{eV}$ and positive values of the real part of $\epsilon_{e f f}$ in the low energy region. Indeed, SEM images (Fig. 2) indicate a surface granular structure with no clear presence of isolated particles. In any case, only the sample with $d_{\text {mass }}=12.5 \mathrm{~nm}$ presents optical constants close to the bulk material values. Samples with smaller thickness present larger optical losses in the infrared range, requiring of Gaussian oscillators in this spectral region. Such behavior has been previously observed in $\mathrm{Au}[24]$ and $\mathrm{Ag}[23,27]$ ultrathin films and is attributed to the collective resonances of closely located nanoparticles that appear around the percolation treshold, where the system morphology transforms from wellseparated nanoparticles to a compact film. This observation indicates that the film becomes more compact as $d_{\text {mass }}$ increases. Indeed, the ratio $d_{\text {mass }} / d_{\text {eff }}$ (Table 1 ) is close to 1 for the thicker samples and smaller for the one with $d_{\text {mass }}=5 \mathrm{~nm}$. This is additionally supported by the observed growth of $\omega_{p}$ with $d_{\text {mass }}$, indicating larger electronic density [28] and the decrease of $\gamma$ with $d_{\text {mass }}$, suggesting a longer electron free path in the film as it becomes more compact. 

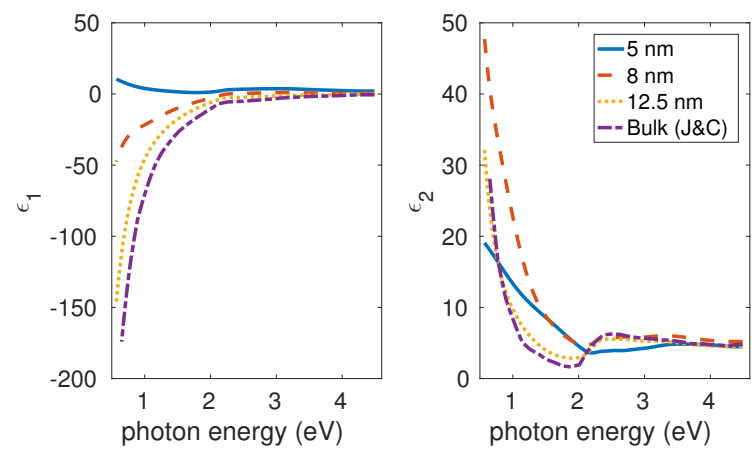

Figure 1: Real (left) and imaginary (right) effective dielectric function of $\mathrm{Cu}$ island films deposited at $T_{s}=\mathrm{RT}$ and with different mass thicknesses.
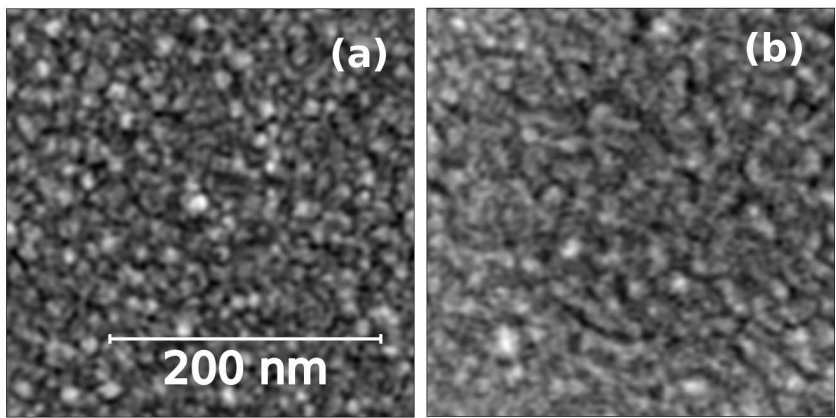

Figure 2: SEM plain view of the sample surface for $\mathrm{Cu}$ island films with 12.5 (left) and $5 \mathrm{~nm}$ (right) deposited at $T_{s}=\mathrm{RT}$. Scale bar for all the images is shown in (a). 


\begin{tabular}{cccccccc}
$d_{\text {mass }}(\mathrm{nm})$ & $T_{s}\left({ }^{\circ} \mathrm{C}\right)$ & $d_{\text {eff }}(\mathrm{nm})$ & $\omega_{p}(\mathrm{meV})$ & $\gamma(\mathrm{eV})$ & $\omega_{s p}(\mathrm{eV})$ & $R_{S E M}(\mathrm{~nm})$ & $\% \mathrm{Cu} / \% \mathrm{Cu}_{2} \mathrm{O}$ \\
\hline 5 & $\mathrm{RT}$ & 7.2 & 1.20 & 365 & - & - & $0 / 100$ \\
5 & 75 & 7.4 & - & - & 0.94 & $18 \pm 8$ & - \\
5 & 135 & 7.3 & - & - & 1.18 & $38 \pm 14$ & - \\
5 & 200 & 9.1 & - & - & 1.28 & $51 \pm 20$ & - \\
8 & $\mathrm{RT}$ & 8.3 & 5.13 & 247 & - & - & $42 / 58$ \\
8 & 75 & 9.7 & 4.86 & 405 & - & $34 \pm 21$ & - \\
8 & 135 & 10.4 & - & - & 0.94 & $39 \pm 15$ & - \\
8 & 200 & 11.4 & - & - & 1.01 & $40 \pm 17$ & $35 / 65$ \\
12.5 & $\mathrm{RT}$ & 13.2 & 7.23 & 98 & - & - & $80 / 20$
\end{tabular}

Table 1: Investigated samples, described by their mass thickness and substrate temperature values, and parameters determined from the analysis of spectroscopic ellipsometric $\left(d_{e f f}, \omega_{p}, \gamma, \omega_{s p}\right)$, SEM (average particle size from plain view images, $\left.R_{S E M}\right)$ and XRD $\left(\% \mathrm{Cu} / \% \mathrm{Cu}_{2} \mathrm{O}\right)$ measurements. The parameters $\omega_{p}$ and $\gamma$ are reported for those samples showing a Drude term in the effective dielectric response while $\omega_{s p}$ is listed for samples showing a well-defined localized surface plasmon resonance.

Next, the role of substrate temperature during deposition, $T_{s}$, is investigated. Fig. 3 shows $\epsilon_{\text {eff }}$ of $\mathrm{Cu}$ island films with $d_{\text {mass }}=8 \mathrm{~nm}$ grown at different $T_{s}$. The samples deposited at lower $T_{s}$ show a metallic behavior while those deposited at higher $T_{s}$ present a well-defined plasmon resonance in the near infrared range. This behaviour is explained by the enhancement of the island growth mechanism as $T_{s}$ increases: evaporated ad-atoms have larger diffusivity on heated substrates and preferentially bind to already deposited metal clusters rather than wet the substrate. It should be noticed that the ratio $d_{\text {mass }} / d_{\text {eff }}$ and $\gamma$ increase and $\omega_{p}$ decreases with $T_{s}$, indicating the reduction of film compactness. These observations are supported by SEM images (Fig. 4), that illustrate the continuous variation from a nearly-compact 
film to a distribution of isolated particles growing in size $\left(R_{S E M}\right.$ in Table 1) as $T_{s}$ increases.
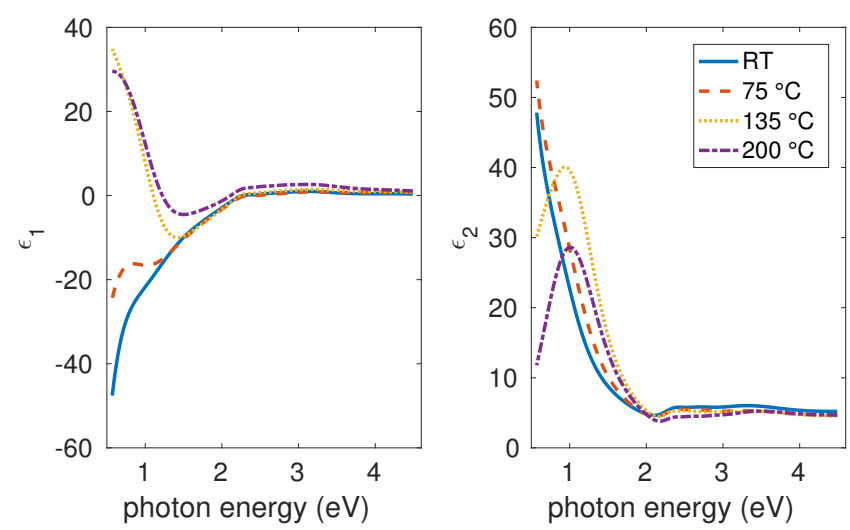

Figure 3: Real (left) and imaginary (right) effective dielectric function of $\mathrm{Cu}$ island films with mass thickness $8 \mathrm{~nm}$ and deposited at different substrate temperatures.

The peak in the imaginary part of $\epsilon_{e f f}$ associated to the localized surface plasmon resonance ( $\omega_{s p}$ in Table 1$)$ shifts to higher photon energies as $T_{s}$ increases, for both film series with $d_{\text {mass }}=5 \mathrm{~nm}$ and $d_{\text {mass }}=8 \mathrm{~nm}$. This blue shift can be attributed to the increase of the particles oblateness, as suggested by the augment of cross section roundness evidenced in SEM images. Fig. 5 shows the variation of the imaginary part of $\epsilon_{e f f}$, calculated according Eq. 1, when the aspect ratio $b / c$ is varied. We assume $a=\frac{2}{3} \sqrt{b c}$, i.e, the particles are flattened in the plane parallel to the sample surface. For $b \neq c$ (non-oblate particles), the depolarization factors are different and the inplane dielectric function is anisotropic in the case that a preferential orientation of ellipsoids exists. In order to account for a random orientation of the semi-axes $b$ and $c$ in the sample plane, an average depolarization factor is used in Eq. 1 [13]. Literature values are used for the dielectric function of $\mathrm{Cu}$ [5], while the embedding medium is assumed to be a mixture of air and $\mathrm{SiO}_{2}\left(\epsilon_{h}=1.56\right)$. The calculation shows that the plasmon peak follows a blue shift and gets attenuated when the particles become 

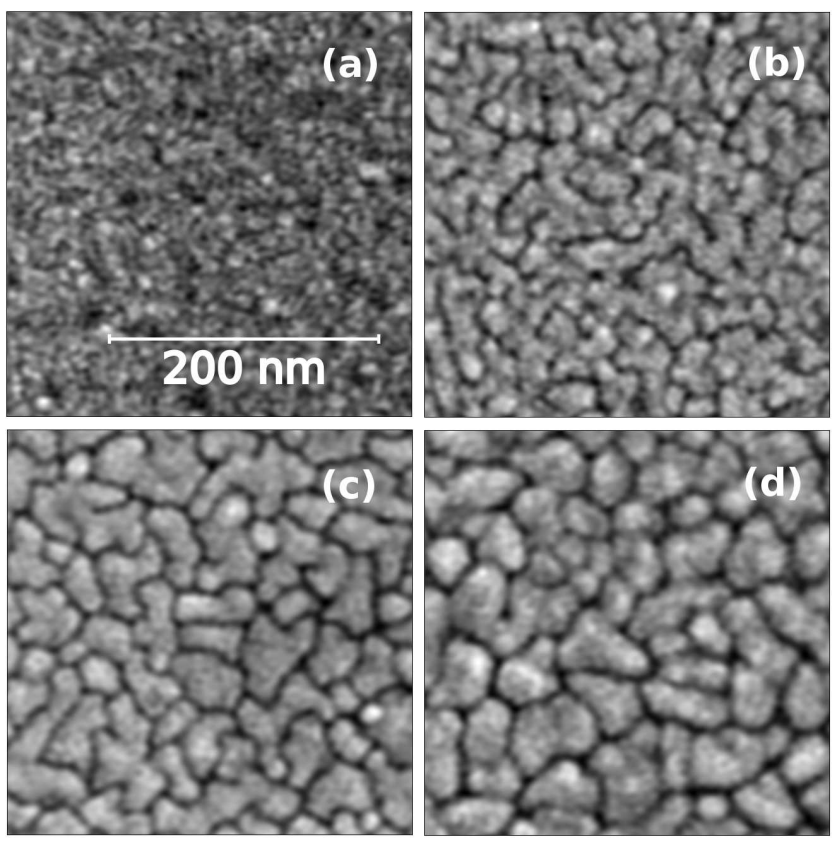

Figure 4: SEM plain view of the sample surface of $\mathrm{Cu}$ island films with $8 \mathrm{~nm}$ mass thickness deposited at different substrate temperatures: room temperature (a), $75^{\circ} \mathrm{C}(\mathrm{b}), 135{ }^{\circ} \mathrm{C}$ (c) and $200{ }^{\circ} \mathrm{C}(\mathrm{d})$. Scale bar for all the images is shown in (a).

more oblate. On the other hand, the observed experimental behavior can be also qualitatively explained by the reduction of particles volume fraction as $T_{s}$ grows. Fig. 6 shows simulation of the imaginary part of $\epsilon_{e f f}$ dependence with $\mathrm{Cu}$ volume fraction, $f$. The observed blue shift and strength decrease of the plasmon resonance with the reduction of $f$ is explained by the weakening of electromagnetic coupling among particles as they become less concentrated [29]. Taking into account the experimental values of the plasmon resonance frequency and the complex morphology of the samples, one can expect that both effects, i.e. increased particle oblateness and volume fraction reduction, contribute to the observed trends. 


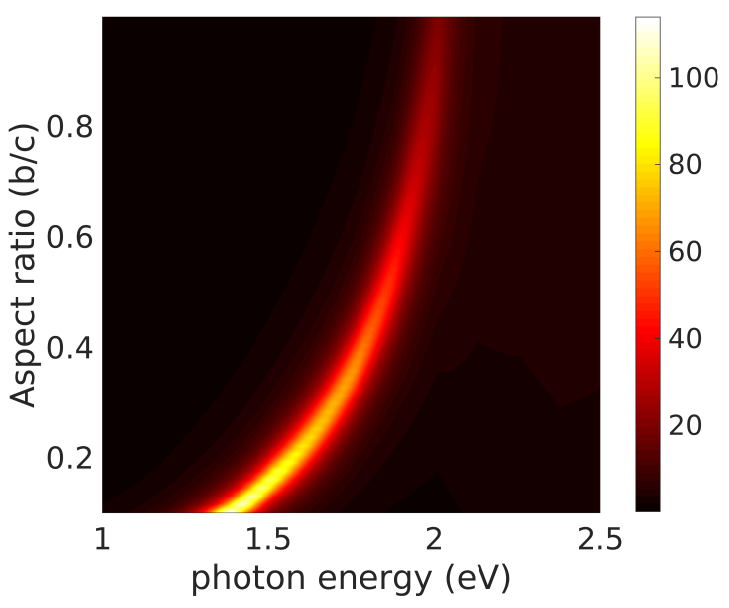

Figure 5: Imaginary part of the effective dielectric function of $\mathrm{Cu}$ island films computed according Eq. 1 assuming a $f=0.5$ and varying the aspect ratio $b / c$ and keeping $a=\frac{2}{3} \sqrt{b c}$ and the particle volume constant. Calculation is based on an average depolarization factor $L_{p}$ in Eq. 1.

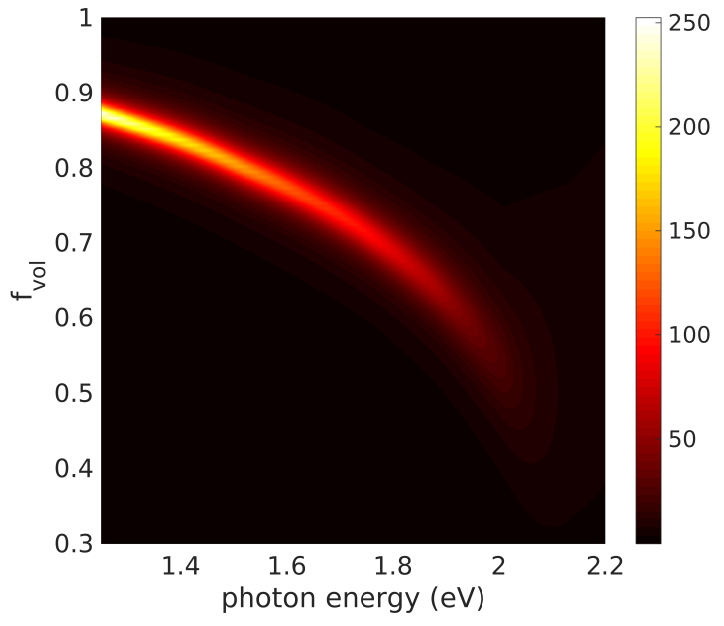

Figure 6: Imaginary part of the effective dielectric function of $\mathrm{Cu}$ island films computed according Eq. 1 , assuming $a / b=2 / 3$ and varying $f$ 


\subsection{Time stability}

The results so far presented suggest that sample deposition at $T_{s}=\mathrm{RT}$ and $d_{\text {mass }}$ smaller than $5 \mathrm{~nm}$ could lead to films consisting of isolated particles. Indeed, transmittance measurements performed just after deposition of a film with $d_{\text {mass }}=2 \mathrm{~nm}$ shows a dip around $1.6 \mathrm{eV}$ (Fig. 7) that can be associated to the excitation of localized surface plasmon resonance in isolated $\mathrm{Cu}$ particles. However, this feature quickly disappears within the first day of exposure at normal atmosphere. The absence of plasmon resonances indicates lack of metal free electrons and suggests the complete oxidization of $\mathrm{Cu}$ islands.

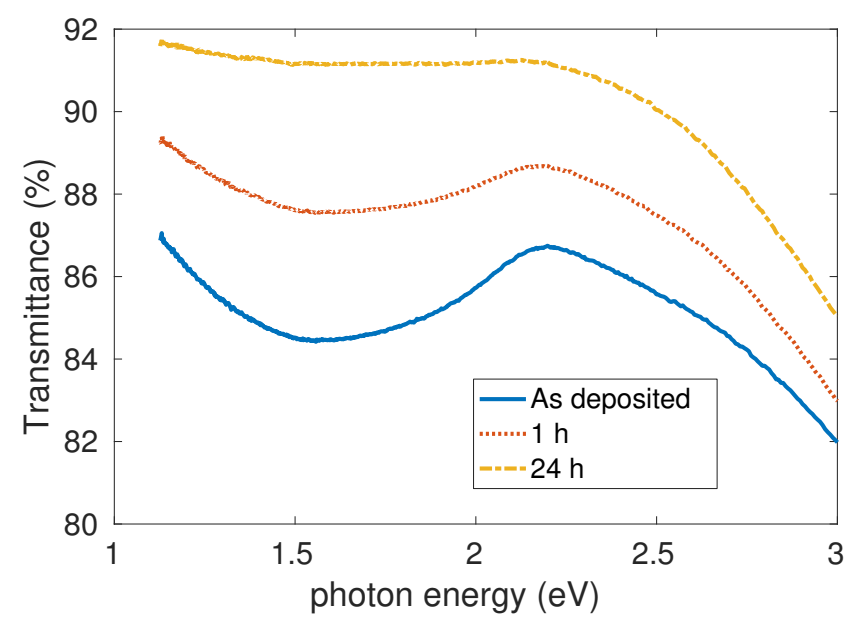

Figure 7: Transmittance spectra of $\mathrm{Cu}$ island films with $d_{\text {mass }}=2 \mathrm{~nm}$ deposited at $T_{s}=\mathrm{RT}$.

The effect of oxidization can be also noticed by investigating the interband transitions appearing in the $\epsilon_{\text {eff }}$ spectra higher energy region. Fig. 8 shows the data retrieved from ellipsometric measurements taken one day and two months after deposition for samples deposited at $T_{s}=\mathrm{RT}$ and having $d_{\text {mass }}=5 \mathrm{~nm}$ and $d_{\text {mass }}=12.5$ $\mathrm{nm}$. One day after deposition both samples show a peak around $2.4 \mathrm{eV}$ that can be attributed to the interband transitions from the valence band $L_{3}$ point to the Fermi 
level in $\mathrm{Cu}$ [30]. The second remarkable peak, centered around $3.5 \mathrm{eV}$, corresponds to the maximum of the imaginary part of the $\mathrm{Cu}_{2} \mathrm{O}$ dielectric function, that is connected to $X_{3} \rightarrow X_{1}$ transitions [31]. The relative strength of the $\mathrm{Cu} / \mathrm{Cu}_{2} \mathrm{O}$ interband transitions is larger for the thicker film, indicating smaller relative oxidization during the first 24 hours. The stability of the thicker film is confirmed by measurements performed after 2 months, showing small changes in $\epsilon_{\text {eff }}$. On the other hand, the second measurement of the film with $d_{\text {mass }}=5 \mathrm{~nm}$ shows lack of $\mathrm{Cu}$ interband transitions and increase of $\mathrm{Cu}_{2} \mathrm{O}$ contribution, suggesting strong film oxidization.

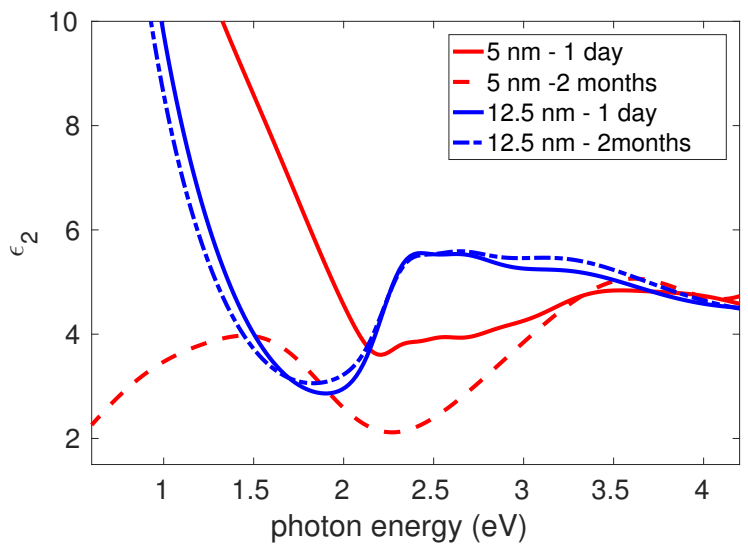

Figure 8: Imaginary part of the $\mathrm{Cu}$ island films effective dielectric function of 5 and $12.5 \mathrm{~nm}$ samples deposited at $T_{s}=\mathrm{RT}$. Data is retrieved from measurements performed one day and two months after deposition.

In order to verify film oxidization and its amount, X-ray diffraction measurements were taken on selected samples two months after deposition (Fig. 9). The diffraction peaks match to $\mathrm{Cu}$ and $\mathrm{Cu}_{2} \mathrm{O}$ phases, which simulated patterns are shown below the experimental spectra. Note a considerable broadening of the XRD peaks as a consequence of the small crystallite size and grazing geometry. Taking into account the scattering cross sections of $\mathrm{Cu}$ and $\mathrm{Cu}_{2} \mathrm{O}$, one can estimate the volume phase 
composition $\left(\% \mathrm{Cu} / \% \mathrm{Cu}_{2} \mathrm{O}\right.$ in Table 1$)$ from the diffraction peak intensities. The thinner the film, the larger the relative oxidization is, in agreement with the results of ellipsometric analysis. However, oxidization appears to be related not only to $d_{\text {mass }}$ but also to the film compactness, as shown by the larger amount of $\mathrm{Cu}_{2} \mathrm{O}$ in the film with $d_{\text {mass }}=8 \mathrm{~nm}$ and $T_{s}=200{ }^{\circ} \mathrm{C}$ compared to the film with same $d_{\text {mass }}$ and deposited keeping the substrate at room temperature. Indeed, less compact films are expected to be more prone to oxidization, as they have larger effective surface for the process to take place. A final remark on the analysis of XRD is that the particle size could be, in principle, estimated from the peak widths using the Scherrer formula. However, the obtained values (4-5 nm) are underestimated and hardly comparable to $R_{S E M}$ due to the instrumental contribution to line broadening characteristic of grazing incidence geometry, the highly anisotropic islands shape and the possibility that the $\mathrm{Cu}$ particles consist of numerous crystallites.

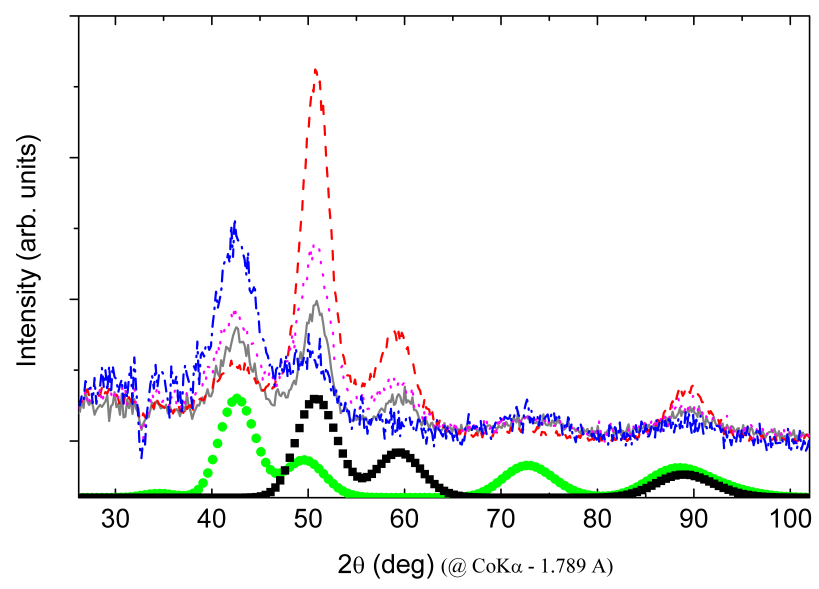

Figure 9: X-ray diffraction scans for $\mathrm{Cu}$ island films (from the highest to the lowest intensity at $\left.50^{\circ}\right): 12.5 \mathrm{~nm}$ deposited at $T_{s}=\mathrm{RT}, 8 \mathrm{~nm}$ deposited at $T_{s}=\mathrm{RT}, 8 \mathrm{~nm}$ deposited at at $T_{s}=200^{\circ} \mathrm{C}$ and $5 \mathrm{~nm}$ deposited at $T_{s}=\mathrm{RT}$. Reference data for $\mathrm{Cu}$ (black squares) and $\mathrm{Cu}_{2} \mathrm{O}$ (green circles) are included at the bottom. Contribution from $\mathrm{SiO}_{2}$ layer has been removed. 
Previous theoretical investigations on $\mathrm{Cu}-\mathrm{Cu}_{2} \mathrm{O}$ core-shell particles have shown that the plasmon resonance monotonically shifts towards lower photon energies as the oxide shell grows [12]. In principle, the same trend could be expected with sample aging, due to the increase of oxidization. However, the experimental results show a more complex behaviour. Fig. 10 shows the localized suface plasmon resonance frequencies, corresponding to transmittance spectra dips, measured at different time intervals for the samples with $d_{\text {mass }}=5 \mathrm{~nm}$. During the first days, aging of the samples result in the expected red shift. Afterwards, the plasmon resonance starts shifting to higher photon energies. For more compact films this turning point appears to happen sooner. Notice that for the sample deposited at $T_{s}=\mathrm{RT}$, during the first 24 hours no transmittance minimum was evidenced in the measured spectral range and once the plasmon peak clearly appeared in the measurements, no red-shift was observed at all.

According to the simulations discussed above (Fig. 5), reshaping of the particles, leading to a long-term increase of their oblateness or sphericity, could be a plausible explanation for the observed trend. Generally, dewetting of ultrathin films requires an annealing process [32] but it may occur in metal island films just by aging [33]. In order to verify possible dewetting and particle reshaping in the films, SEM images were taken on the sample with $d_{\text {mass }}=5 \mathrm{~nm}$ and deposited at $T_{s}=75{ }^{\circ} \mathrm{C}$ two months after deposition. The comparison of the sample surface one week and two months after deposition is shown in Fig. 11. No significant changes on particle morphology that could support this hypothesis were detected. On the contrary, aging resulted in a more compact film as a consequence of the oxide overlayer growth, i.e., the incorporation of oxygen made the film more compact and thicker. Indeed, ellipsometric measurements taken at later times confirmed a slight increase of $d_{\text {eff }}$.

Upon oxidization, a fraction of $\mathrm{Cu}$ atoms become part of the shell and the $\mathrm{Cu}$ 


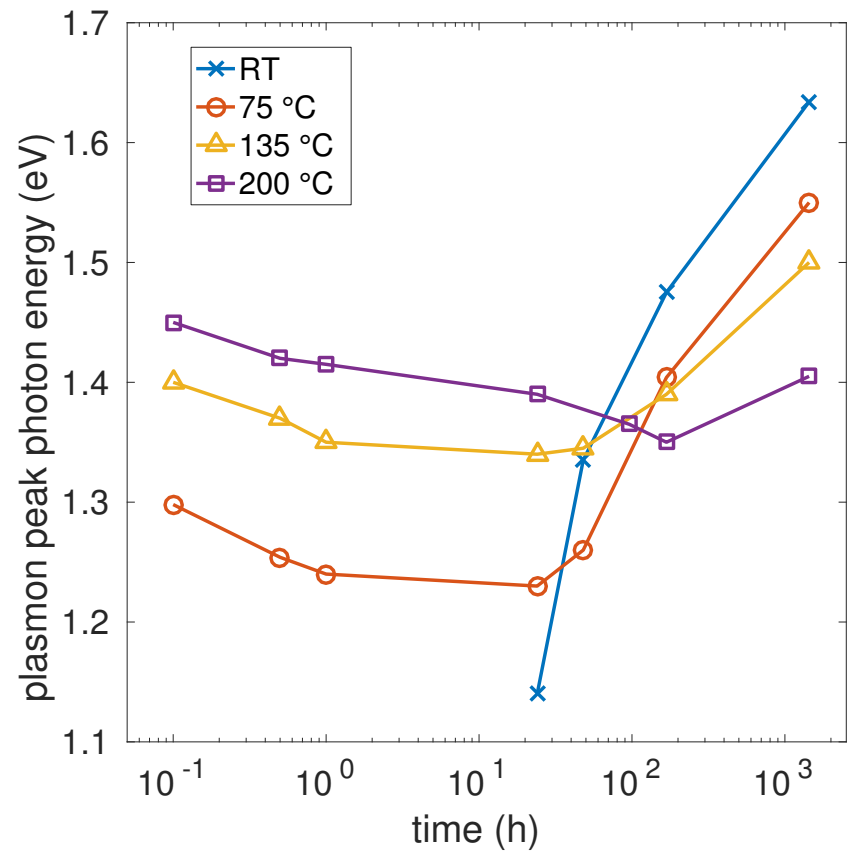

Figure 10: Time evolution of the plasmon peak position (transmittance minimum) for $\mathrm{Cu}$ island films with $5 \mathrm{~nm}$ mass thickness deposited at different substrate temperatures. 
core diminishes its size. This leads to a reduction of the overall volume fraction of $\mathrm{Cu}$ in the film that results in weaker electromagnetic coupling among particles and blue shift of the plasmon resonance, as discussed earlier (Figure 6). Therefore, there is a competition between a red-shift generated by the presence of oxide and a blue-shift caused by lower volume concentration. It appears that the red-shift resulting from the increase of the dielectric function of the medium surrounding the particles dominates during the first stages of oxidization. When the shell is thick enough, the medium surrounding the particles can be considered as primarily $\mathrm{Cu}_{2} \mathrm{O}$ and the plasmon resonance shift saturates. After this point, additional oxidization results only in reduced electromagnetic coupling that appears to dominate further plasmon resonance shift. Films having a morphology consisting of isolated particles have low interparticle coupling from the beginning and the turning point can take place at a long time. On the contrary, samples with morphology around or above the percolation threshold have much stronger interparticle interaction and the reduction of its strength upon oxidization can be noticed in the spectra at an early stage. These explanations are supported by the calculation of $\epsilon_{\text {eff }}$ assuming a constant value of volume particle concentration $f$, but reducing the relative $\mathrm{Cu}$ volume concentration, $f_{C u}$. Therefore, we replace $f$ by $f_{C u}$ in Eq. 1 and $\epsilon_{h}$ is approximated as an average of the air- $\mathrm{SiO}_{2}$ medium $\left(\epsilon_{\mathrm{Air}-\mathrm{SiO}_{2}}=1.56\right)$ and $\mathrm{Cu}_{2} \mathrm{O}$, i.e.:

$$
\epsilon_{h}=\frac{(1-f) \epsilon_{{\mathrm{Air}-S i O_{2}}}+f\left(1-f_{\mathrm{Cu}}\right) \epsilon_{\mathrm{Cu}_{2} \mathrm{O}}}{(1-f)+f\left(1-f_{C u}\right)} .
$$

Simulations shown in Fig. 12 and 13 verify that the turning point between red and blue shift of the plasmon resonance indeed takes place at lower $\mathrm{Cu}$ concentration, i.e., at a more advanced oxidization state, for less compact films. This non-trivial trend can cause, for instance, that samples that initially did not show a clear surface plasmon signature (see the film with $d_{\text {mass }}=5 \mathrm{~nm}$ deposited at $T_{s}=\mathrm{RT}$ ) can later 

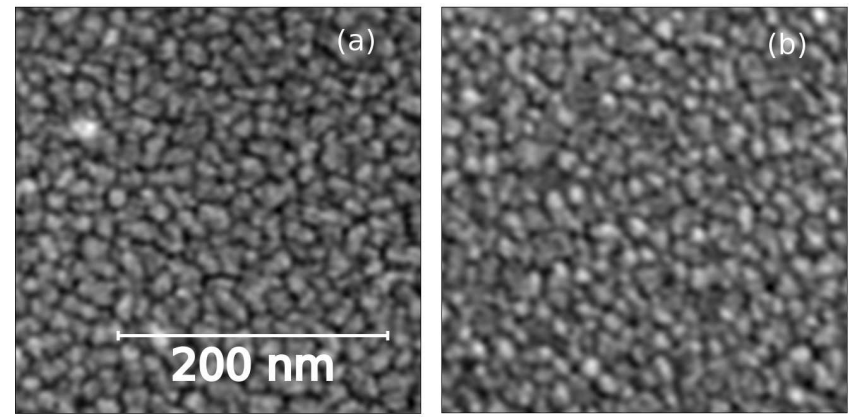

Figure 11: SEM picture of the $\mathrm{Cu}$ island films with mass thickness of $5 \mathrm{~nm}$ and deposited at $75^{\circ} \mathrm{C}$ 2 days (left) and 2 months (right) after deposition. Scale bar for all the images is shown in (a).

present a plasmon peak $(\approx 1.5 \mathrm{eV}$ in Fig. 8) at photon energies higher than for the plasmon resonances of samples that at the beginning had an isolated particle geometry.

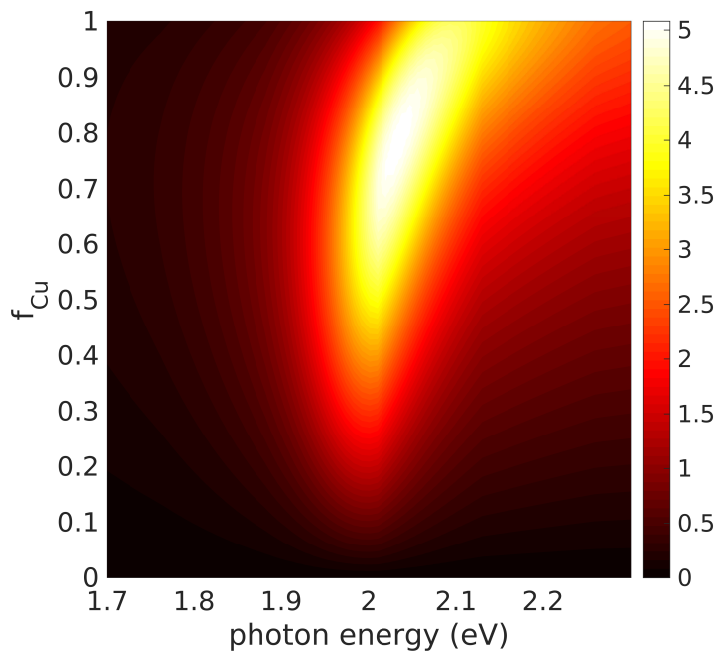

Figure 12: Imaginary part of the effective dielectric function of oxidized $\mathrm{Cu}$ island films computed according Eq. 1, for $f=0.33$ and varying the overall volume fraction of $\mathrm{Cu}, f_{C u}$. 


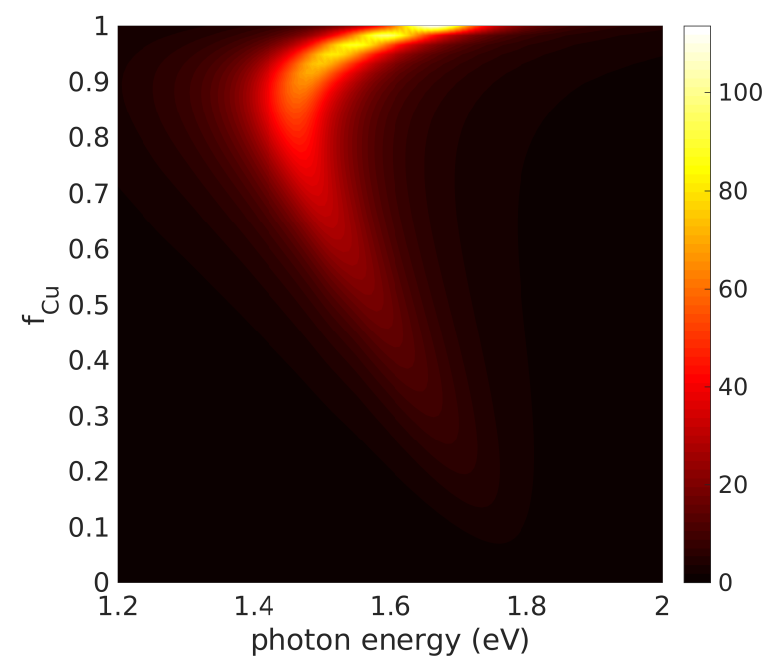

Figure 13: Imaginary part of the effective dielectric function of oxidized $\mathrm{Cu}$ island films computed according Eq. 1, for $f=0.75$ and varying the overall volume fraction of $\mathrm{Cu}, f_{C u}$

\section{Conclusions}

The optical properties of $\mathrm{Cu}$ island films deposited by electron beam evaporation have been investigated and correlated to the morphological and compositional characteristics of the films. The variation of easily controllable parameters like mass thickness and substrate temperature allow to broadly modify the optical response of the film. The well-established thin film technolgy approach used for sample fabrication and the highly tunable localized surface plasmon resonance peak make $\mathrm{Cu}$ island films attractive candidates to be considered for plasmonic applications.

The time evolution of films optical properties is dominated by oxidization upon normal atmosphere exposition, resulting in large modifications in thinner films, while thicker films remain more stable. The evolution of the plasmon peak appears to be driven by two competing trends: an initial red-shift resulting from the increase of the surrounding dielectric matrix and a blue shift at a later stage that is consequence 
of the reduced electromagnetic coupling among particles. Overall, the obtained results bring new insights that can be useful to understand the stability of plasmonic properties of $\mathrm{Cu}$ island films.

\section{Acknowledgements}

This work was supported by the Ministry of Environment and Energy, the Ministry of Science and Education, the Environmental Protection and Energy Efficiency Fund and the Croatian Science Foundation under the project Plasmonic Alternative Materials for Solar Energy Conversion" (PKP-2016-06-4469) in the total amount of $1074000 \mathrm{HRK}$.

\section{References}

[1] S. A. Maier, Plasmonics: fundamentals and applications, Springer, 2007.

[2] E. Ozbay, Plasmonics: merging photonics and electronics at nanoscale dimensions, Science 311 (5758) (2006) 189-193.

[3] P. R. West, S. Ishii, G. V. Naik, N. K. Emani, V. M. Shalaev, A. Boltasseva, Searching for better plasmonic materials, Laser \& Photonics Reviews 4 (6) (2010) 795-808.

[4] E. Palik, Handbook of Optical Constants of Solids, Academic Press handbook series, Academic Press, 1998.

[5] P. B. Johnson, R.-W. Christy, Optical constants of the noble metals, Physical review B 6 (12) (1972) 4370.

[6] G. V. Naik, V. M. Shalaev, A. Boltasseva, Alternative plasmonic materials: beyond gold and silver, Advanced Materials 25 (24) (2013) 3264-3294. 
[7] T. Ghodselahi, M. Vesaghi, A. Shafiekhani, A. Baghizadeh, M. Lameii, XPS study of the $\mathrm{Cu} @ \mathrm{Cu}_{2} \mathrm{O}$ core-shell nanoparticles, Applied Surface Science 255 (5) (2008) 2730-2734.

[8] G. H. Chan, J. Zhao, E. M. Hicks, G. C. Schatz, R. P. Van Duyne, Plasmonic properties of copper nanoparticles fabricated by nanosphere lithography, Nano Letters 7 (7) (2007) 1947-1952.

[9] Q.-C. Sun, Y. Ding, S. M. Goodman, H. H. Funke, P. Nagpal, Copper plasmonics and catalysis: role of electron-phonon interactions in dephasing localized surface plasmons, Nanoscale 6 (21) (2014) 12450-12457.

[10] V. Kravets, R. Jalil, Y.-J. Kim, D. Ansell, D. Aznakayeva, B. Thackray, L. Britnell, B. Belle, F. Withers, I. Radko, et al., Graphene-protected copper and silver plasmonics, Scientific reports 4 (2014) 5517.

[11] M. D. Susman, Y. Feldman, A. Vaskevich, I. Rubinstein, Chemical deposition and stabilization of plasmonic copper nanoparticle films on transparent substrates, Chemistry of Materials 24 (13) (2012) 2501-2508.

[12] O. Peña-Rodríguez, U. Pal, Effects of surface oxidation on the linear optical properties of $\mathrm{Cu}$ nanoparticles, Journal of the Optical Society of America B 28 (11) (2011) 2735-2739.

[13] M. Held, O. Stenzel, S. Wilbrandt, N. Kaiser, A. Tünnermann, Manufacture and characterization of optical coatings with incorporated copper island films, Applied optics 51 (19) (2012) 4436-4447.

[14] E. Dobierzewska-Mozrzymas, P. Biegański, Optical properties of discontinuous copper films, Applied optics 32 (13) (1993) 2345-2350. 
[15] O. Stenzel, A. Macleod, Metal-dielectric composite optical coatings: underlying physics, main models, characterization, design and application aspects, Advanced Optical Technologies 1 (6) (2012) 463-481.

[16] J. Sancho-Parramon, V. Janicki, H. Zorc, On the dielectric function tuning of random metal-dielectric nanocomposites for metamaterial applications, Optics express 18 (26) (2010) 26915-26928.

[17] R. Singer, A. Leitner, F. Aussenegg, Structure analysis and models for optical constants of discontinuous metallic silver films, JOSA B 12 (2) (1995) 220-228.

[18] T. Jensen, R. Van Duyne, S. Johnson, V. Maroni, Surface-enhanced infrared spectroscopy: a comparison of metal island films with discrete and nondiscrete surface plasmons, Applied Spectroscopy 54 (3) (2000) 371-377.

[19] P. Royer, J. Goudonnet, R. Warmack, T. Ferrell, Substrate effects on surfaceplasmon spectra in metal-island films, Physical Review B 35 (8) (1987) 3753.

[20] V. A. Markel, Introduction to the Maxwell Garnett approximation: tutorial, Journal of the Optical Society of America A 33 (7) (2016) 1244-1256.

[21] C. F. Bohren, D. R. Huffman, Absorption and scattering of light by small particles, John Wiley \& Sons, 2008.

[22] T. Yamaguchi, H. Takahashi, A. Sudoh, Optical behavior of a metal island film, JOSA 68 (8) (1978) 1039-1044.

[23] J. Sancho-Parramon, V. Janicki, H. Zorc, Tuning the effective dielectric function of thin film metal-dielectric composites by controlling the deposition temperature, Journal of Nanophotonics 5 (1) (2011) 051805. 
[24] M. Hövel, B. Gompf, M. Dressel, Dielectric properties of ultrathin metal films around the percolation threshold, Physical Review B 81 (3) (2010) 035402.

[25] T. V. Amotchkina, M. K. Trubetskov, A. V. Tikhonravov, V. Janicki, J. SanchoParramon, H. Zorc, Comparison of two techniques for reliable characterization of thin metal-dielectric films, Applied optics 50 (33) (2011) 6189-6197.

[26] M. Lončarić, J. Sancho-Parramon, H. Zorc, Optical properties of gold island films - a spectroscopic ellipsometry study, Thin Solid Films 519 (9) (2011) 29462950.

[27] A. J. de Vries, E. S. Kooij, H. Wormeester, A. A. Mewe, B. Poelsema, Ellipsometric study of percolation in electroless deposited silver films, Journal of applied physics 101 (5) (2007) 053703.

[28] C. Kittel, P. McEuen, P. McEuen, Introduction to solid state physics, Vol. 8, Wiley New York, 1996.

[29] J. Sancho-Parramon, Surface plasmon resonance broadening of metallic particles in the quasi-static approximation: a numerical study of size confinement and interparticle interaction effects, Nanotechnology 20 (23) (2009) 235706.

[30] H. Ehrenreich, H. Philipp, Optical properties of $\mathrm{Ag}$ and $\mathrm{Cu}$, Physical Review 128 (4) (1962) 1622.

[31] T. Ito, T. Kawashima, H. Yamaguchi, T. Masumi, S. Adachi, Optical properties of $\mathrm{Cu}_{2} \mathrm{O}$ studied by spectroscopic ellipsometry, Journal of the Physical Society of Japan 67 (6) (1998) 2125-2131.

[32] C. V. Thompson, Solid-state dewetting of thin films, Annual Review of Materials Research 42 (2012) 399-434. 
[33] H. Hieber, Aging properties of gold layers with different adhesion layers, Thin Solid Films 37 (3) (1976) 335-343. 